\title{
Chronotype differences in circadian rhythms of temperature, melatonin, and sleepiness as measured in a modified constant routine protocol
}

This article was published in the following Dove Press journal:

Nature and Science of Sleep

3 November 2009

Number of times this article has been viewed

Leon Lack

Michelle Bailey

Nicole Lovato

Helen Wright

School of Psychology, Flinders University, Adelaide, South Australia, Australia
Correspondence: Leon Lack School of Psychology, Flinders University, GPO Box 2100, Adealide, SA 500I, Australia

Tel $+6 \mid 8$ 820I 239|

Fax +61882013877

Email leon.lack@flinders.edu.au

\begin{abstract}
Evening chronotypes typically have sleep patterns timed 2-3 hours later than morning chronotypes. Ambulatory studies have suggested that differences in the timing of underlying circadian rhythms as a cause of the sleep period differences. However, differences in endogenous circadian rhythms are best explored in laboratory protocols such as the constant routine. We used a 27-hour modified constant routine to measure the endogenous core temperature and melatonin circadian rhythms as well as subjective and objective sleepiness from hourly 15-minute sleep opportunities. Ten (8f) morning type individuals were compared with 12 (8f) evening types. All were young, healthy, good sleepers. The typical sleep onset, arising times, circadian phase markers for temperature and melatonin and objective sleepiness were all 2-3 hours later for the evening types than morning types. However, consistent with past studies the differences for the subjective sleepiness rhythms were much greater (5-9 hours). Therefore, the present study supports the important role of subjective alertness/sleepiness in determining the sleep period differences between morning and evening types and the possible vulnerability of evening types to delayed sleep phase disorder.
\end{abstract}

Keywords: chronotype, constant routine, circadian rhythms, sleep propensity, subjective sleepiness

\section{Introduction}

Individual differences in the timing of the sleep/wake schedule are usually described in terms of chronotype as measured, for example, by a morningness-eveningness questionnaire. ${ }^{1}$ Morning-type individuals have earlier sleep-wake schedules, earlier diurnal peaks of alertness and performance and earlier sleep propensity rhythms than evening-type individuals. ${ }^{2-6}$

It has long been suggested that chronotype differences arise from differences in the timing of their endogenous circadian rhythms that regulate the daily variations in a number of biological and behavioral processes. ${ }^{7-12}$ Since early studies found significant differences between morning- and evening-type individuals in the timing of the core temperature and melatonin rhythms, some authors have suggested that the behavioral and sleep timing differences are produced by timing or phase differences in their endogenous biological rhythms. ${ }^{3,4,13}$ This notion was supported by the finding of different phase angles of entrainment between morning and evening types. Although morning types had earlier temperature rhythms, they slept at a relatively later temperature rhythm phase than evening types. ${ }^{14-16}$ In other words there was less difference in selected bed periods than in circadian phase. This would suggest that the circadian rhythm differences are driving the sleep period differences in opposition

submit your manuscript $\mid$ www.dovepress.con 
to the social need for morning and evening types to sleep at more similar conventional times.

However, much of this research did not control the potential masking of endogenous rhythm differences by effects imposed from the timing of the sleep/wake cycle and associated environmental changes (eg, light/dark variation) or did not collect data over a full circadian cycle. ${ }^{17}$ The constant routine protocol was designed to unmask the endogenous circadian rhythm by controlling confounding factors such as physical activity, sleep, and light exposure. ${ }^{18,19}$ Those studies employing constant routines or other laboratorybased methods have also found significant circadian rhythm phase differences between morning and evening types but have not found larger differences in endogenous circadian timing than self-selected sleep periods..$^{20-22}$

Based on the premise that the rhythms with the largest timing differences between chronotypes are likely contributors to the other rhythm differences, ${ }^{4}$ the comparability of endogenous biologic rhythm differences and typical sleep periods leaves the etiology of the sleep period differences unclear. ${ }^{21}$ The only rhythm variable in some studies showing larger phase differences between morning and evening types is that of subjective sleepiness/alertness. As early as $1980,{ }^{3}$ and later in ambulatory studies with only daytime measurements, ${ }^{6}$ highest alertness in the morning for morning types and highest alertness in the evening for evening types suggested a greater phase difference in alertness than their temperature rhythms. Vidacek and colleagues ${ }^{4}$ tested across a 24-hour period and found a two-hour difference between chronotypes in temperature rhythms but a significant four-hour difference in the alertness rhythms. They were the first to suggest the possibility that the alertness rhythm may be endogenous and contribute to the temperature and sleep period timing differences. Kerkhof and Van Dongen used continuous rectal temperature recording and frequent alertness measures over two weeks of ambulatory monitoring. ${ }^{20}$ The differences between morning and evening types in timing of sleep period, actigraphy rhythms, and core temperature were all very similar (2.5-3.0 hours). However, the alertness rhythms differed by six hours. They then collected the same measures in a constant routine protocol with very similar results. The importance of the difference between chronotypes in this dimension of subjective sleepiness needs to be confirmed.

A measure conceptually related to subjective sleepiness is that of objective sleepiness or sleep propensity as measured by sleep latency or amount of sleep obtained in brief sleep opportunities. This variable has received very little attention despite the fact that it is a measure of the ability to initiate and sustain sleep, an important determiner of the timing of objective sleep. Using an ultrashort sleep/wake cycle of 20 minutes, Lavie and Segal found one phase marker of the sleep propensity rhythm, the 'sleep gate,' to be later in three evening types than the five morning types. ${ }^{5}$ However, their methodology was not able to measure other biological circadian rhythms.

Both subjective sleepiness and sleep propensity are likely to influence when an individual chooses to go to bed or to arise in the morning. This, in turn, may affect their circadian phase indirectly through cessation and resumption of retinal light stimulation. No study to our knowledge has included both of these important measures. Therefore, the present study evaluated the rhythms of sleep propensity and subjective sleepiness as well as biological circadian rhythms of core temperature and melatonin activity in morning and evening types.

Like the individual differences present in all biological and behavioral characteristics, the differences between morning and evening types probably represents normal variation of chronotype between individuals. ${ }^{13}$ However, the extremes of the morningness-eveningness dimension are associated with advanced and delayed sleep phase disorder and are classified as circadian rhythm disorders. ${ }^{23}$ However, it is often difficult to effectively and permanently alleviate advanced or delayed sleep phase disorder using only chronobiologic treatments aimed at phase shifting or retiming the endogenous circadian rhythm. ${ }^{24,25}$ This suggests that the sleep timing difference between morning- and evening-types is not simply a result of the endogenous circadian rhythm phase differences, but also involves lifestyle and psychological factors in the choice of bedtime and arising time..$^{21,25,26}$ Therefore, furthering the understanding of the etiology of sleep/wake timing differences between morning- and evening-type individuals may have implications for the treatment of circadian rhythm disorders. The aim of the present study was to explore the differences between morning and evening types in the timing of sleepiness rhythms as well as endogenous biological circadian rhythms.

\section{Methods}

\section{Participants}

The study included 22 undergraduate psychology students aged between 23-45 years who were recruited from Flinders University. Subjects were selected from an initial sample of 313 respondents to the morningness-eveningness questionnaire. ${ }^{1}$ From the frequency distribution of these 
313 participants, those with the most outlying scores were invited to participate in the laboratory study. This included participants who were classified as morning types with scores ranging from 64 to 77 or evening types whose scores ranged from 16 to 41 .

The morning type group included two males and eight females (mean age $=27.40$, standard deviation $[S D]=10.34$ ). Within this group two subjects were definite morning types with scores $>70$ and eight moderate morning types with scores ranging from 64 to 69 . The evening type group consisted of four males and eight females (mean age $=23.75$, $\mathrm{SD}=7.01)$. There were nine definite evening types with scores $<31$ and three moderate evening types with scores ranging from 36 to 41 .

All participants were self-reported good sleepers. This was confirmed by the typical week of sleep diary and wrist actigraphy calculated sleep just prior to the laboratory session. Inclusion criteria consisted of mean sleep onset latency $<30$ minutes, wake time after sleep onset $<30$ minutes, total sleep time between seven and nine hours, satisfactory daytime functioning, and body mass index between 20 and 30 .

None had clinical symptoms of any sleep disorder. None were taking any medication known to affect their sleep nor did they regularly consume excessive amounts of alcohol ( $>2$ standard drinks per day) or caffeine ( $>300 \mathrm{mg}$ estimated caffeine per day). The study received approval from the Flinders University Social and Behavioural Research Ethics Committee and participants were given a gratuity of A \$100 for participation.

\section{Test instruments}

Core body temperature was measured using a series 400 flexible rectal temperature probe (Yellow Springs Instruments, Yellow Springs, OH, USA). The temperature probe was self-inserted $10 \mathrm{~cm}$ into the rectum and then connected to an opto-isolation interface box and recorded continuously during the study.

Melatonin activity was assessed from urine voidings collected every two hours. Urine volume was recorded to the nearest $1 \mathrm{ml}$ and a $5 \mathrm{ml}$ aliquot was then mixed with boracic acid and frozen. The urine samples were analyzed by the Department of Obstetrics and Gynaecology at the University of Adelaide for the major melatonin metabolite concentration using the 6-sulphatoxymelatonin radioimmunoassay. ${ }^{27}$ Since urinary 6-sulphatoxymelatonin levels are a reliable indicator of plasma melatonin, ${ }^{28}$ for simplicity we will refer to the 6-sulphatoxymelatonin measures as melatonin activity throughout this report.
Sleep propensity was assessed during a 15-minute sleep opportunity every hour. At the start of the laboratory session participants were fitted with electroencephalogram (CZ, OZ, A1), electrooculogram, and electromyogram electrodes for standard recording. Throughout the laboratory session electrode impedances were checked periodically and maintained at less than $5 \mathrm{~K}$ Ohms. Sleep was determined consistent with conventional criteria. ${ }^{29}$ The sleep propensity measure was the total amount of sleep (in minutes) of any stage obtained in the 15-minute opportunity. Once sleep was initiated it invariably continued for the rest of the trial. The sleep propensity measure was essentially the inverse of sleep latency.

Subjective sleepiness was assessed hourly, immediately prior to each 15-minute sleep opportunity using the Stanford Sleepiness Scale (SSS). ${ }^{30}$ Participants were asked to indicate which number (1-7) best described how they were currently feeling, higher scores reflected higher levels of subjective sleepiness.

\section{Prior to laboratory sessions}

Participants were required to maintain their typical bed and wake times for seven days prior to the laboratory session including the night before the laboratory session. Sleep diaries were used to indicate times of lights-out, sleep onset, and final wake time. Gähwiler Z80 actigraphy monitors (SinghMedical, Stata, Switzerland) were worn on the nondominant wrist throughout this period and confirmed the diary data.

\section{Laboratory sessions}

Morning-type participants entered the laboratory at approximately 1930 hours and evening-type participants entered the laboratory two hours later at approximately 2130 hours. This two-hour time difference was implemented as morning types had their sleep period two hours earlier than the evening types as has been consistently shown in research. ${ }^{13,31,32}$ Knowledge of clock time from mobile phones, watches, clocks or experimenters was prohibited throughout their stay in the laboratory as it may have influenced subjective feelings of sleepiness or sleep propensity.

Participants were then shown to their separate bedrooms and prepared for PSG recording. The rectal thermistor was self-inserted to the $10 \mathrm{~cm}$ tactile indicator on the thermistor lead. Morning-type participants were then confined to bed by 2045 hours and evening-type participants were confined to bed by 2245 hours to begin the testing regime. Participants remained in bed for a 27-hour protocol. The bedrooms were sound attenuated and ambient temperature was controlled at $20{ }^{\circ} \mathrm{C}$ throughout the laboratory session. Light intensity at the 
participant's head from an overhead incandescent light was maintained at 40 lux between sleep-opportunity trials. The low intensity and long wavelengths of this light should not have produced any melatonin suppression or circadian phase change. ${ }^{33-35}$ To control for the thermogenic effect of food intake, small snacks of approximately equal caloric value and a $200 \mathrm{ml}$ glass of water or diluted juice were provided every two hours.

The modification of the constant routine procedure in the present study included hourly tests of the amount of sleep recorded in a 15-minute sleep opportunity as the measure of sleep propensity. Over 27 consecutive hours, these 15-minute sleep opportunities alternated with 45-minute enforced wakeful periods. Every hour the lights were turned out for 15 minutes and subjects were asked to attempt sleep. Participants were awoken by the researcher at the end of the 15-minute sleep opportunity by opening their bedroom door and calling their name. During the 45 minutes of each hour when participants were required to stay awake, they were permitted to engage in quiet activities such as watching DVDs, reading, studying or listening to music while maintaining a near supine position. Immediately prior to each sleep opportunity participants completed the SSS. Urine samples were collected every two hours at least 10 minutes before a sleep trial.

\section{Overview of statistical analyses}

Temperature data was first averaged into 30-minute bins over 26 hours (discarding the first hour in bed). A best-fit twocomponent (24-hour fundamental and 12-hour harmonic) cosine function was calculated for the 52 consecutive temperature data points for each participant and circadian parameter estimates of temperature minimum (Tmin) and maximum (Tmax) were obtained from the fitted curve. ${ }^{10,15,36}$ The average variance accounted for by the curve fits to temperature data was $71.3 \%$. Two-component cosine functions were also fitted to individual variations of subjective sleepiness (SSS) and sleep propensity measures in order to determine maximum and minimum phase timings in these measures for both groups.

From the raw 6-sulphatoxymelatonin data, a value that was $100 \%$ above baseline values was established. The time of dim light melatonin activity onset (DLMO) was determined by interpolating between the first data value that consistently exceeded this value and the previous two-hourly value. A sixth term polynomial curve was fitted to the melatonin activity data for each participant to estimate the time of the maximum melatonin activity. Robust melatonin activity curves were obtained from all participants with group average 24-hour totals within the normal range for these relatively young adult participants. ${ }^{37}$ The average variance accounted for by the complex curve fits was $69.71 \%$.

\section{Results}

\section{Preliminary analysis}

Table 1 shows the preliminary analyses conducted to assess whether differences in morningness-eveningness predict differences in the timing of sleep periods. T-tests indicated that typical sleep onset times and final wake up times were significantly earlier by about two and a half hours for MT compared to ET participants. There was no difference in total sleep time between groups.

\section{Circadian rhythm timing}

Figure 1 illustrates the timing of the group means for the typical sleep periods in shaded horizontal bars and mean sleep propensity maximum (SPmax) and minimum times (SPmin). Also indicated are the circadian phase markers of core temperature minimum (down arrows) and dim light melatonin activity onsets (DLMO). All of these measures were about 2-3 hours different between morning and evening types. Also illustrated in Figure 1 are the means for subjective sleepiness maximum (SSmax) and subjective sleepiness minimums (SSmin) for the two groups. These differences between evening and morning types are about six and nine hours, respectively, and were statistically significant $(P<0.001)$. Thin dashed lines are drawn connecting equivalent phase markers of the two groups. The flatter slopes connecting subjective sleepiness phases for the two groups illustrates the greater differences in timing of subjective sleepiness than in the other circadian and sleep propensity rhythms.

Table 2 shows the group means (SD) for the morning types and evening types of all circadian and sleepiness measures and the statistically independent sample $t$-tests of

Table I Mean sleep onset times, final wake-up times, and total sleep time for morning (MT) and evening type (ET) participants in the week prior to the laboratory session showing the difference and $t$-test results of the difference

\begin{tabular}{lllll}
\hline & MT & ET & $\begin{array}{l}\text { Difference } \\
\text { hours }\end{array}$ \\
\hline Sleep onset time & $2324(\mathrm{I} .07)$ & $0159(\mathrm{I} .28)$ & 2.58 & $4.9^{*}$ \\
Final wake-up time & $0748(\mathrm{I} .0)$ & $1020(1.59)$ & 2.53 & $4.29^{*}$ \\
Total sleep time (hrs) & $8.15(0.64)$ & $8.20(0.73)$ & 0.05 & n.s. \\
\hline
\end{tabular}

Note: $* P<0.001$. 


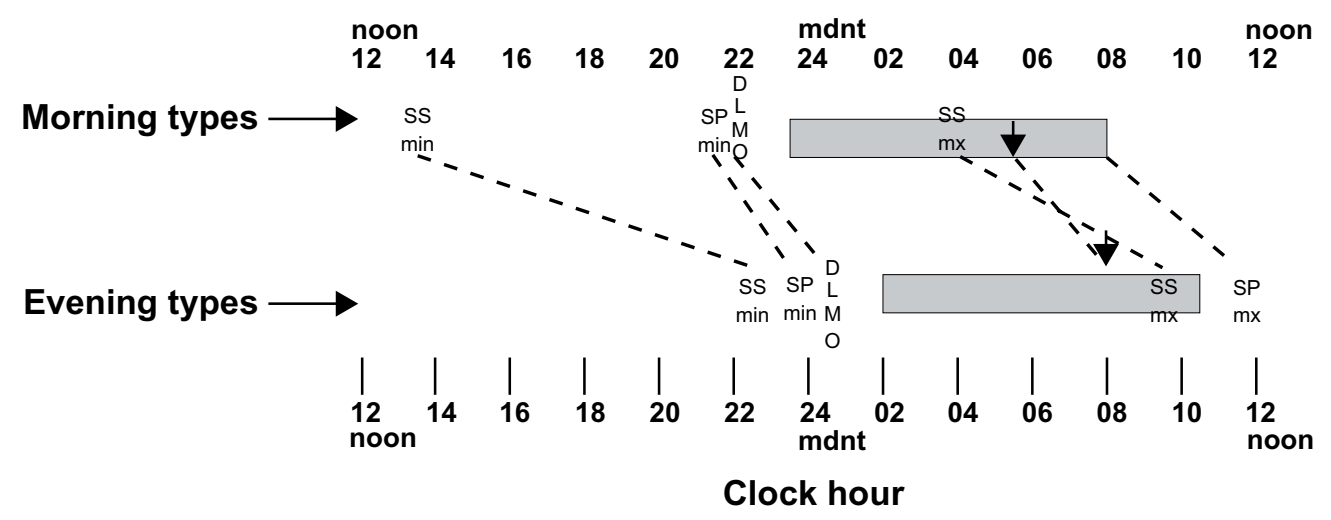

Figure I The mean times for the morning and evening type groups of their typical sleep period (horizontal shaded bars), of their subjective sleepiness minimum (SSmn) and maximum (SSmx), objective sleep propensity maximum (the maximum sleep in the I5-minute hourly sleep opportunities, SPmx and sleep propensity minimum (SPmin), dim light urinary melatonin onset (DLMO), and core temperature minimum phase,Tmin (down arows). The same phase markers for the two groups are connected with thin dashed lines so that the slopes of the connecting lines can indicate the relative differences between the two groups. It can be seen that the circadian and sleep timings are all about 2-3 hours later for the evening types. However, the subjective sleepiness phases show the greatest differences between groups. The morning types feel least sleepy in the early afternoon and the evening types least sleepy or most alert in the late evening, only a few hours before their typical bedtimes. The morning types then feel most sleepy before their Tmin (down arrow) but the evening types feel most sleepy after their Tmin at about their typical wake time.

the group differences. The phase markers for all dependent variables (core temperature, melatonin activity, sleep propensity, and subjective sleepiness) were earlier for the morning-type than the evening-type participants by at least two hours. Only the sleep propensity minimum phase difference did not reach statistical significance. The greater group differences in the subjective sleepiness rhythm phases are documented in Table 2. The minimum subjective sleepiness of the morning types occurs close to the middle of the day while for the evening types it occurs over nine hours later in the late evening. This is consistent with the evening types' reported feelings of alertness and preference for activities in the evening only a few hours before their intended bed time. The mean subjective sleepiness maximum for the morning types occurs before Tmin in the middle of their typical sleep period whereas for the evening types it occurs more than five hours later and after their Tmin close to their typical wake-up time.

Apart from the subjective sleepiness rhythms all the other rhythms and typical sleep times have consistent internal phase relationships within groups and are all consistently later in the evening types by about 2-3 hours. The subjective sleepiness rhythm was the exception with relatively early phase times for the morning types and late phase times for the evening types. For example, the phase difference between core body temperature minimum and maximum subjective sleepiness (Tmin-SSmx) was significantly more positive $(\mathrm{t}(19)=2.19$, $P<0.05)$ for the morning types $(\mathrm{M}=1.35$ hours, $\mathrm{SD}=4.07)$ than the evening types $(\mathrm{M}=-1.91$ hours, $\mathrm{SD}=2.06)$. Therefore, the subjective sleepiness rhythm was, relative to the other rhythms, delayed in the evening types.

\section{Discussion}

Because the extreme ends of the chronotype dimension eveningness-morningness can lead to the circadian rhythm disorders of delayed and advanced sleep phase, this study aimed to provide a better understanding of the causes of sleep period differences between chronotypes. In order to measure unmasked circadian rhythms of core body temperature and melatonin activity as well as sleep propensity and

Table $2 T$-tests for the differences between the phase markers of morning type (MT) and evening type (ET) participants

\begin{tabular}{|c|c|c|c|c|c|}
\hline Phase marker & & MT clock time (SD) & ET clock time (SD) & Difference (hours) & $t$ value \\
\hline Core temperature & Tmin & $0513(1.62)$ & 0728 (I.87) & 2.25 & $2.88 * *$ \\
\hline \multirow[t]{2}{*}{ Melatonin } & Max & $0420(0.93)$ & $0620(0.7)$ & 2.00 & $2.55^{* *}$ \\
\hline & DLMO & $2148(1.4)$ & $2436(0.84)$ & 2.80 & $5.42 * * *$ \\
\hline \multirow[t]{2}{*}{ Sleep propensity } & Min & $2128(3.5)$ & $2334(2.88)$ & 2.10 & 1.5 \\
\hline & Max & $0759(3.83)$ & I I 20 (4.74) & 3.35 & $\mathrm{I} .79 *$ \\
\hline \multirow[t]{2}{*}{ Subjective sleepiness } & Min & 1318 (3.28) & $2229(3.58)$ & 9.18 & $6.02 * * *$ \\
\hline & Max & $0352(1.45)$ & $0922(4.33)$ & 5.50 & 4.II*** \\
\hline
\end{tabular}

Notes: $* P<0.05 ; * * P<0.1 ; * * * P<0.001$

Abbreviations: DLMO, dim light melatonin onset; SD, standard deviation. 
subjective sleepiness rhythms we used a modified constant routine protocol. We reasoned that if there were greater differences between chronotypes in circadian rhythm timing than typical sleep periods it would suggest that circadian phase differences drive behavioral differences. On the other hand, if sleep period differences were greater than rhythm differences, it would suggest the circadian rhythms are simply following lifestyle choices and subsequent differential exposure to zeitgebers such as light/dark cycles. If all differences were about the same, it would not be possible to attribute differential causal direction.

We found almost all the phase differences between the morning and evening types in this study to be in the order of 2-3 hours. The differences between groups in core temperature and melatonin activity rhythms were not greater than the typical sleep onset and wake-up times and thus does not support the notion that the circadian rhythm differences produce the sleep period timing differences. The sleep propensity rhythms, indicating the ability to initiate sleep, were also different by the same amount and thus cannot be necessarily identified as a cause of the sleep period differences.

However, the exception to this pattern of results was in the measure of subjective sleepiness. The mean maximum subjective sleepiness times were 5.5 hours different between groups and minimum sleepiness or maximum alertness was over nine hours different between groups. Furthermore, maximum sleepiness occurred significantly earlier relative to the temperature rhythm phase for the morning types than evening types. In another demographically similar sample of young, healthy good sleepers, but of intermediate chronotype, maximum subjective sleepiness virtually coincided with the core temperature minimum. ${ }^{12}$ In contrast the maximum subjective sleepiness of the morning types in the present study preceded their core temperature minimum by 1.35 hours but in the evening types it followed their temperature minimum by 1.91 hours. In the morning types peak alertness occurred about a third of the way through their typical wake period and maximum sleepiness occurred about midpoint in their typical sleep period. Therefore, their subjective sleepiness rhythm would seem to be conducive for them maintaining a stable timing of bed time and arising time.

However, the evening types' peak alertness occurred only 3.5 hours before their typical bed time and maximum sleepiness occurred only an hour before their typical arising time. This relatively late timing of the subjective sleepiness rhythm would predispose the evening types to delay bedtime and arising time. With work and social obligations requiring a forced arising time during the normal working week this is likely to result in some sleep loss during the week. Taking the opportunity when available (eg, on the weekend) to catch up on this lost sleep mainly by sleeping later in the morning has been shown to result in a delay of circadian rhythms which will exacerbate the already late timed circadian rhythms of evening types. ${ }^{25,38-40}$ Therefore, the delayed timing of the subjective sleepiness rhythm in the evening types would increase the likelihood of them developing delayed sleep phase disorder.

The results of the present study reinforce the findings of earlier studies with respect to subjective alertness/sleepiness circadian rhythms showing a much greater phase difference between morning and evening types than the typical sleep period and biological circadian rhythms. This suggests that relatively delayed subjective alertness/sleepiness rhythms in evening types may be driving their delay of sleep period. In turn it may be the case that the delay in sleep period timing and consequential timing differences in retinal light exposure then delays the circadian rhythms of the evening types.

Alternatively, the relatively early timed subjective sleepiness rhythm of the morning types may be predisposing them to the choice of relatively early bed times and arising times. This possibility, however, is not supported by the relatively late timed sleep propensity minimum and maximums in relation to their typical sleep period. The morning types, similar to the evening types, fall asleep least readily in the later evening consistent with the 'wake-maintenance zone' about eight hours before their core temperature minimum. ${ }^{10,41,42}$ Therefore, morning types would experience some difficulty initiating sleep at bed times more than two hours before their typical bed times and such experiences would encourage them to maintain a consistent bed time rather than drift earlier.

Recent studies have found evidence suggesting that evening types appear to accumulate homeostatic sleep drive (Process S) more slowly during wakefulness than do morning types and dissipate it more slowly during sleep. ${ }^{43-45}$ Net subjective sleepiness and sleep propensity are functions of both homeostatic sleep drive and circadian sleep drive. However, if homeostatic sleep drive accumulates more slowly in the evening types, it would tend to produce relative delays of sleepiness rhythm markers. This putative mechanism may thus be contributing to the greater differences between chronotypes at least in subjective sleepiness.

Another possible biological difference predisposing evening types to a later circadian phase is differences in endogenous period length. A longer period length ( $>24$ hours) would produce a tendency to phase delay. A forced de-synchrony 
study found that greater eveningness was significantly related to longer intrinsic period length. ${ }^{17}$ Evening types had intrinsic period lengths of about 24.3 hours compared to about 24.1 hours in the more morning types. More recently a timeisolation, free-running study compared an extreme evening type, who also met the criteria for delayed sleep phase disorder, with three control intermediate chronotypes. ${ }^{46}$ With selfselected bed periods the evening type showed a consistently longer period length of 25.4 hours compared to 24.4 hours for the controls. It seems likely that these long period lengths (more than nine SD above the mean) do not reflect true intrinsic circadian period lengths. It would seem more likely that in their self-selected bed time protocol that the longer sleep/wake period length of the evening type arose from a greater period length of the subjective sleepiness rhythm. In turn the more rapid delay of bed times would have led to the delay of evening light exposure and resultant longer temperature rhythm period length in the extreme evening type. Future research should attempt to unconfound these factors potentially contributing to the delayed circadian phase of evening types as well as the more extreme delayed sleep phase disorder cases.

It can be argued that by opting to include a measure of sleep propensity involving hourly sleep opportunities of up to a maximum of 15 minutes sleep every hour we have degraded the purity of the constant routine protocol and our core temperature measurements. However, from a previous similar protocol comparing a constant routine with a subsequent modified routine with regularly timed brief sleep opportunities similar to the present study we found no differences in measured mean temperature minimums and a high correlation in the temperature nadirs from both routines. ${ }^{10}$ Furthermore, the melatonin activity measures would not have been affected by periodic naps in the low illumination conditions of the present study, ${ }^{33-35}$ and they showed the same order of phase differences between chronotypes as the core temperature measures. Therefore we are confident that the temperature minimum values in the present study are valid measures of the endogenous temperature minimum.

There are clinical implications for the treatment of delayed sleep phase disorder and the prevention of its development in evening chronotypes. The fact that circadian rhythms and sleep initiation ability are timed significantly later in evening than morning types would suggest that chronobiologic treatment (eg, morning bright light and early evening melatonin administration) should be helpful in stabilizing and phase advancing the rhythms of evening types thus preventing the development of delayed sleep phase disorder. This would be particularly important if longer intrinsic period lengths in more extreme evening types constantly predisposed them to circadian phase delay.

However, the most salient clinical implication in the present study arises from the greater timing differences in subjective sleepiness rhythm that would predispose evening types to choose bed times later than their ability to initiate sleep. Both the morning and evening types show sleep propensity minimums (longest sleep latencies) in their 'wakemaintenance zones' only a few hours before their typical bed time as predicted from their core temperature rhythms. This zone is followed shortly by the dim light melatonin onset and an increased ability to initiate sleep. However, at this point subjective sleepiness would still be lower in the evening types than the morning types who have reached their minimum much earlier in the day and who will reach their maximum sleepiness significantly earlier after the dim light melatonin onset. Thus the evening types should be able to initiate sleep at a comparable circadian phase to the morning types but they simply will not feel as sleepy and will tend to delay their bedtime. Therefore, cognitive/behavior therapies aimed at encouraging a somewhat earlier than desired bed time and discouraging sleeping late into the morning should be a useful adjunct to the normal chronobiologic therapies in the treatment of delayed sleep phase disorder or its prevention in more extreme evening types. ${ }^{25}$

\section{Disclosures}

The authors report no conflicts of interest in this work.

\section{References}

1. Horne JA, Östberg O. A self-assessment questionnaire to determine morningness-eveningness in human circadian rhythms. Int J Chronobiol. 1976;4(2):97-110.

2. Horne JA, Brass CG, Pettitt AN. Circadian performance differences between morning and evening 'types'. Ergononmics. 1980;23(1):29-36.

3. Kerkhof GA, Korving HJ, Willemse-v.d. Geest HM, Rietveld WJ Diurnal differences between morning-type and evening-type subjects in self-rated alertness, body temperature and the visual and auditory evoked potential. Neurosci Lett. 1980;16(1):11-15.

4. Vidacek S, Kaliterna L, Rodosevic-Vidacek B, Folkard S. Personality differences in the phase of circadian rhythms: a comparison of morningness and extraversion. Ergonomics. 1988;31(6):873-888.

5. Lavie P, Segal S. Twenty-four-hour structure of sleepiness in morning and evening persons investigated by ultra short sleep-wake cycle. Sleep . 1989;12(6):522-528.

6. Natale V, Cicogna P. Circadian regulation of subjective alertness in morning and evening 'types'. Person Individ Diff. 1996;20(4):491-497.

7. Kleitman N. Sleep and Wakefulness. Chicago, IL: University of Chicago Press; 1963.

8. Aschoff J. Circadian rhythms in man: a self-sustained oscillator with an inherent frequency underlies human 24-hour periodicity. Science. 1965;148(3676):1427-1432.

9. Czeisler CA, Weitzman ED, Moore-Ede MC, Zimmerman JC, Kronauer RS. Human sleep: its duration and organization depend on its circadian phase. Science. 1980;210(4475):1264-1267. 
10. Lack LC, Lushington $\mathrm{K}$. The rhythms of human sleep propensity and core body temperature. J Sleep Res. 1996;5(1):1-11.

11. Czeisler CA, Duffy JF, Shanahan TL, et al. Stability, precision, and near-24-hour period of the human circadian pacemaker. Science. 1999;284(5423):2177-2181.

12. Gradisar M, Lack L. Relationships between the circadian rhythms of finger temperature, core temperature, sleep latency, and subjective sleepiness. J Biol Rhythms. 2004;19(2):157-163.

13. Horne JA, Ostberg O. Individual differences in human circadian rhythms. Biol Psychol. 1977;5(3):179-190.

14. Duffy J, Dijk DJ, Hall E, Czeisler C. Relationship of endogenous circadian melatonin and temperature rhythms to self-reported preference for morning or evening activity in young and older people. J Investig Med. 1999;47(3):141-150.

15. Baehr E, Revelle W, Eastman C. Individual differences in the phase and amplitude of the human circadian temperature rhythm: with an emphasis on morningness-eveningness. J Sleep Res. 2000;9(2):117-127.

16. Liu X, Uchiyama M, Shibui K, et al. Diurnal preference, sleep habits, circadian sleep propensity and melatonin rhythm in healthy human subjects. Neurosci Lett. 2000;280(3):199-202.

17. Duffy J, Rimmer D, Czeisler C. Association of intrinsic circadian period with morningness-eveningness, usual wake time, and circadian phase. Behav Neurosci. 2001;115(4):895-899.

18. Czeisler CA, Brown EN, Ronda JM, Kronauer RE, Richardson GS, Freitag WO. A clinical method to assess the endogenous circadian phase (ECP) of the deep circadian oscillator in man. Sleep Res. 1985;14:295.

19. Duffy JF, Dijk DJ. Getting through to circadian oscillators: why use constant routines? J Biol Rhythms. 2002;17(1):4-13.

20. Kerkhof G, Van Dogen H. Morning-type and evening-type individual differ in the phase position of their endogenous circadian oscillator. Neurosci Lett. 1996;218(3):153-156.

21. Waterhouse J, Folkhard S, Dongen H, et al. Temperature profiles, and the effect of sleep on them, in relation to morningness-eveningness in healthy female subjects. Chronobiol Int. 2001;18(2):227-247.

22. Goulet G, Mongrain V, Desrosiers C, Paquet J, Dumont M. Daily light exposure in morning-type and evening-type individuals. J Biol Rhythms. 2007;22(2):151-158.

23. American Academy of Sleep Medicine. The International Classification of Sleep Disorders. 2nd ed. Westchester, IL: American Academy of Sleep Medicine; 2005.

24. Regestein QR, Monk T. Delayed sleep phase syndrome: A review of its clinical aspects. Am J Psychiatry. 1995;152(4):602-608.

25. Lack LC, Wright HR, Bootzin RR. Delayed sleep-phase disorder. Sleep Med Clin. 2009;4(2):229-239.

26. Wittmann M, Dinich J, Merrow M, Roenneberg T. Social jetlag: misalignment of biological and social time. Chronobiol Int. 2006;23(1-2): 497-509.

27. Aldhous ME, Arendt J. Radioimmunoassay for 6-sulfatoxymelatonin in urine using an iodinated tracer. Ann Clin Biochem. 1988;25(Pt 3): 298-303.
28. Graham C, Cook MR, Kavet R, Sastre A, Smith DK. Prediction of nocturnal plasma melatonin from morning urinary measures. $J$ Pineal Res. 1998;24(4):230-238.

29. Rechtschaffen A, Kales A. A Manual of Standardized Terminology, Techniques and Scoring Systems for Sleep Stages in Human Subjects. Los Angeles, CA: Brain Information Service/Brain Research Institute; 1968.

30. Hoddes E, Zarcone V, Smythe H, Phillips R, Dement W. Quantification of sleepiness: A new approach. Psychophysiology. 1973;10(4):431-436.

31. Clodore M, Foret J, Benoit O. Diurnal variations in the subjective and objective measures of sleepiness: The effects of sleep reduction and circadian type. Chronobiol Int. 1986;3(4):255-263.

32. Mongrain V, Dumont M. Increased homeostatic response to behavioral sleep fragmentation in morning types compared to evening types. Sleep. 2007;30(6):773-780.

33. Lewy AJ. The dim light melatonin onset, melatonin assays and biological rhythm research in humans. Biol Signals Recept. 1999;8(1-2):79-83.

34. McIntire IM, Norman TR, Burrows GD, Armstrong SM. Human melatonin suppression by light is intensity dependent. J Pineal Res. 1989;6(2):149-156.

35. Wright HR, Lack LC. Effect of light wavelength on suppression and phase delay of the melatonin rhythm. Chronobiol Int. 2001;18(5):801-808.

36. Barrett J, Lack L, Morris M. The sleep-evoked decrease of body temperature. Sleep. 1993;16(2):93-99.

37. Kennaway DJ, Lushington K, Dawson D, Lack L, van den Heuvel C, Rogers N. Urinary 6-sulfatoxymelatonin excretion and aging: New results and a critical review of the literature. J Pineal Res. 1999;27(4): 210-220.

38. Yang CM, Spielman A. The effect of a delayed weekend sleep pattern on sleep and morning functioning. Psychol Health. 2001;16(6):715-725.

39. Burgess HJ, Eastman CI. A late wake time phase delays the human dim light melatonin rhythm. Neurosci Lett. 2006;395(3):191-195.

40. Taylor A, Wright HR, Lack LC. Sleeping-in on the weekend delays circadian phase and increases sleepiness the following week. Sleep Biol Rhythms. 2008;6(3):172-179.

41. Strogatz SH, Kronauer RE, Czeisler CA. Circadian pacemaker interferes with sleep onset at specific times each day: role in insomnia. Am J Physiol. 1987;253(1):R172-R178.

42. Dijk DJ, Czeisler CA. Paradoxical timing of the circadian rhythm of sleep propensity serves to consolidate sleep and wakefulness in humans. Neurosci Lett. 1994;166(1):63-68.

43. Taillard J, Philip P, Coste O, Sagaspe P, Bioulac B. The circadian and homeostatic modulation of sleep pressure during wakefulness differs between morning and evening chronotypes. J Sleep Res. 2003;12(4):275-282.

44. Mongrain V, Carrier J, Dumont M. Circadian and homeostatic sleep regulation in morningness-eveningness. J Sleep Res. 2006;15(2):162-166.

45. Schmidt C, Collette F, Leclercq Y, et al. Homeostatic sleep pressure and responses to sustained attention in the suprachiasmatic area. Science. 2009;324(5926):516-519

46. Campbell SS, Murphy PJ. Delayed sleep phase disorder in temporal isolation. Sleep. 2007;30(9):1225-1228.
Nature and Science of Sleep

\section{Publish your work in this journal}

Nature and Science of Sleep is an international, peer-reviewed, open access journal covering all aspects of sleep science and sleep medicine, including the neurophysiology and functions of sleep, the genetics of sleep, sleep and society, biological rhythms, dreaming, sleep disorders and therapy, and strategies to optimize healthy sleep. The journal welcomes

\section{Dovepress}

original research, clinical \& epidemiological studies, reviews \& evaluations, case reports and extended reports. The manuscript management system is completely online and includes a very quick and fair peerreview system, which is all easy to use. Visit http://www.dovepress.com/ testimonials.php to read real quotes from published authors. 